\title{
Inteligencia artificial y gobernanza de datos en las administraciones públicas: reflexiones y evidencias para su desarrollo
}

\section{Artificial intelligence and data governance in public administration: reflections and evidence for its development}

Miquel Salvador Serna

Universitat Pompeu Fabra (España)

ORCID: https://orcid.org/0000-0001-8287-0779

miquel.salvador@upf.edu

\begin{abstract}
NOTA BIOGRÁFICA
Profesor titular del Departamento de Ciencias Políticas y Sociales de la Universidad Pompeu Fabra. Doctor en Ciencia Política y de la Administración y Máster en Teoría Política y Social por la Universidad Pompeu Fabra, Licenciado en Ciencias Políticas y Sociología y Máster en Gestión Pública por la Universidad Autònoma de Barcelona. Ha realizado estancias de investigación en el Interdisciplinary Institute of Management de la London School of Economics and Political Science y ha sido Jean Monnet Fellow en el European University Institute de la Unión Europea en Florencia. Su actividad de docencia e investigación se centra en los ámbitos de gestión de recursos humanos, capacidades institucionales, análisis y evaluación de políticas públicas, y en los procesos de transformación digital de las organizaciones públicas. Los resultados de su actividad: https://producciocientifica. upf.edu/CawDOS?id=84701d79f5a7517b\&idioma=es\&tipo=activ.
\end{abstract}

\section{RESUMEN}

El desarrollo y la integración de la Inteligencia Artificial (IA) a nivel transversal en las organizaciones del sector público, trascendiendo de iniciativas puntuales de carácter sectorial, requiere contar con nuevas capacidades. La revisión de diferentes aproximaciones que abordan esta cuestión permite destacar la importancia de los datos y, más concretamente, de su gobernanza en las administraciones públicas. Para profundizar en ello se analizan las diferentes dimensiones de la gobernanza de datos y se identifican cinco componentes para su desarrollo: la estrategia, la arquitectura e infraestructura de datos, la organización (incluyendo la estructura y los procesos), la gestión del talento y las competencias de los profesionales y el modelo de relaciones de la organización con su entorno. A través de la reflexión conceptual y su aplicación a un estudio de caso sobre el Ayuntamiento de Barcelona, con aportes en los diferentes componentes, se destacan aprendizajes y se formulan propuestas para cada uno de ellos. Las conclusiones permiten destacar la necesidad de contar con una estrategia integrada de refuerzo institucional que relacione los diferentes componentes de una gobernanza de datos vinculada al desarrollo de la Inteligencia Artificial en el sector público.

\section{PALABRAS CLAVE}

Inteligencia Artificial; Gobernanza de Datos; Administración Pública.

\section{ABSTRACT}

The development and integration of Artificial Intelligence (AI) at a transversal level in public sector organizations, transcending specific initiatives of a sectoral nature, requires new capabilities. The review 
of different approaches that address this issue highlights the importance of data and, more specifically, its governance in public administrations. To delve into this, the different dimensions of data governance are analyzed and five components are identified for its development: strategy, data architecture and infrastructure, organization (including structure and processes), talent and professionals' competencies' management and the organization's relationship model with its environment. Through conceptual reflection and its application to a case study of the Barcelona City Council, with contributions to the different components, we can highlight learnings and formulate proposals for each of them. The conclusions address the need for an integrated institutional strengthening strategy that relates the different components of data governance linked to the development of Artificial Intelligence in the public sector.

\section{KEYWORDS}

Artificial Intelligence; Data Governance; Public Administration.

\section{SUMARIO}

INTRODUCCIÓN. 1. DISEÑO DE LA INVESTIGACIÓN Y METODOLOGÍA. 2. INTELIGENCIA ARTIFICIAL (IA) Y GOBERNANZA DE DATOS EN EL SECTOR PÚBLICO. 3. PRESENTACIÓN Y ANÁLISIS DE CASO: GOBERNANZA DE DATOS EN EL AYUNTAMIENTO DE BARCELONA. 3.1. EN RELACIÓN A LA ESTRATEGIA. 3.2. EN RELACIÓN A LA ARQUITECTURA DE DATOS Y A LA INFRAESTRUCTURA. 3.3. EN RELACIÓN A LA ORGANIZACIÓN. 3.4. EN RELACIÓN A LA GESTIÓN DEL TALENTO Y LAS COMPETENCIAS. 3.5. EN RELACIÓN AL MODELO RELACIONAL. 4. DISCUSIÓN Y CONCLUSIONES. REFERENCIAS BIBLIOGRÁFICAS.

\section{INTRODUCCIÓN 1}

En el contexto de la denominada Revolución 4.0 y la irrupción de las tecnologías inteligentes (smart technologies) como el internet de las cosas o el blockchain, la Inteligencia Artificial (IA) se apunta como una de las innovaciones disruptivas llamadas a transformar radicalmente el comportamiento de personas y de organizaciones.

La IA ha constituido el objeto de estudio de múltiples disciplinas y que todavía no cuenta con una definición universalmente aceptada (Grosz et al., 2016). En una aproximación amplia, el concepto se asocia a sistemas de computación capaces de recabar datos e información de diferentes fuentes, pensar, aprender y actuar de acuerdo con unos objetivos vinculados a unos algoritmos. A efectos de la investigación que se presenta se ha asociado el concepto de IA al campo de la tecnología y las ciencias computacionales (aunque con aportaciones de otros campos como la biología, la neurociencia, la lingüística, la estadística, entre otros), que incluye una serie de técnicas basadas en algoritmos y máquinas (software y hardware) con capacidad para automatizar actividades, realizar operaciones análogas al aprendizaje y toma de decisiones, y evolucionar con o sin la intervención humana (Castro y New, 2016).

Las organizaciones del sector privado cuentan con una notable trayectoria en el desarrollo y configuración de soluciones basadas en IA para mejorar sus modelos de negocio y de relación con el entorno. Pero todavía no sucede lo mismo en las organizaciones del sector público. Esta demora que no deja de sorprender teniendo en cuenta que la materia prima sobre la que trabaja la IA son los datos y que precisamente las administraciones públicas son organizaciones intensivas en su recolección y gestión. Probablemente ello se deba, entre otras, a que el tipo de tratamiento que se da a este recurso de información no resulta el más apropiado para su optimización a través de la IA y el desarrollo de aplicaciones derivadas. En la medida que los datos contengan errores, sean incompletos, no estén sistematizados y no sean íntegros, bajará la calidad de la información y ello dificultará la producción de resultados fiables en base a ella.

Pero el impulso externo, como el proveniente de organismos internacionales o empresas de consultoría, además de la demanda ciudadana, están propiciando avances y generando oportunidades para incorporar la IA a la actuación de las organizaciones públicas (Informe Intel-ligència artificial: decisions automatitzades a Catalunya, 2020; Desouza, 2018). El surgimiento de soluciones vinculadas a determinados ámbitos secto-

1 El autor agradece a los dos evaluadores anónimos y a los editores la labor de revisión que han realizado y las propuestas de mejora que han aportado, cuyo inestimable valor ha permitido mejorar y enriquecer la versión final del artículo. 
riales (como salud, educación, seguridad, movilidad, entre otros) ofrecen nuevas respuestas a problemas de política pública (Nikitas et al., 2020; Qian Sun y Medaglia, 2019; Allam y Dhunny, 2019; Luckin et al., 2016). Estas respuestas sectoriales adolecen, en muchas ocasiones, de planteamientos centrados exclusivamente en su ámbito o departamento, sin generar sinergias ni apoyarse en una estrategia a nivel corporativo. Ello propicia desarrollos desiguales que en ocasiones dificultan la integración de respuestas ante problemas complejos como los que enfrenta el sector público.

Para consolidar estas iniciativas y facilitar la integración de la IA a nivel corporativo, resulta relevante abordar las dos cuestiones comentadas, la necesidad de contar con datos íntegros y adecuadamente gestionados y la incorporación de una visión integrada de su utilización para reforzar estrategias de IA a nivel corporativo. El presente artículo se propone aportar conceptos, evidencias y reflexiones en esta línea.

Para ello se revisa el debate académico sobre la IA y la gobernanza de datos, aportando una visión integrada que permite definir cinco componentes para su desarrollo: la estrategia, la arquitectura e infraestructura de datos, la organización, la gestión del talento y las competencias de los profesionales y el modelo de relaciones de la organización con su entorno. Además de su definición, se plantea la utilización de dichos componentes a modo de esquema analítico que permite valorar la situación de la gobernanza de datos en una organización. Para complementar la vertiente conceptual y analítica se presenta su aplicación concreta en el estudio de caso del Ayuntamiento de Barcelona, como aporte que permite ilustrar su potencial y ofrecer evidencias concretas de los avances realizados y retos pendientes en la vinculación de la gobernanza de datos y la IA en el sector público.

Además del aporte al debate académico, el artículo ofrece a los responsables de las organizaciones públicas una reflexión y una serie de aprendizajes a partir del estudio de caso del Ayuntamiento de Barcelona. La aplicación del esquema analítico propuesto permite sistematizar su revisión, destacar tanto logros como retos pendientes, y poner en valor la necesidad de contar una estrategia integrada de gobernanza de datos para el desarrollo de la IA en la administración pública.

A nivel de exposición, y siguiendo el hilo argumental expuesto, el artículo se inicia con un primer apartado en el que se presenta el diseño de la investigación y de la metodología planteada, que combina la aproximación conceptual con la definición de un esquema analítico que se aplica a un estudio de caso orientado a la acción (action-oriented case study). En un segundo apartado se presenta el aporte conceptual a partir de la revisión del debate académico sobre la IA y la gobernanza de datos, que culmina con la definición de los cinco componentes clave que se plantean para su desarrollo conjunto. En el tercer apartado se utiliza el esquema analítico basado en los cinco componentes para abordar el estudio de caso del Ayuntamiento de Barcelona, elaborado a partir de un proyecto de colaboración que permitió combinar diferentes técnicas (desde el análisis documental y de datos, realización de reuniones con un equipo de seguimiento y realización de entrevistas a informadores clave) y aportar evidencias sobre los conceptos clave que se abordan. El apartado final se dedica a la discusión y conclusiones, donde se destacan los aportes realizados a nivel conceptual y analítico y, a partir de los mismos, se argumenta la necesidad de desarrollar una estrategia integral de gobernanza de datos para sentar las nuevas capacidades que deberían facilitar el desarrollo de la IA en las organizaciones del sector público.

\section{DISEÑO DE LA INVESTIGACIÓN Y METODOLOGÍA}

El objetivo del artículo es aportar reflexión y evidencias, a nivel conceptual y aplicado, sobre la relación entre la gobernanza de datos y la IA en las organizaciones del sector público.

A nivel más concreto se plantea indagar sobre cómo los componentes de gobernanza de datos resultan críticos para el desarrollo integral -más allá del sectorial- de la IA en las administraciones públicas.

Para ello se propone una primera aportación en clave de reflexión conceptual sobre la vinculación entre IA y datos, incorporando el concepto de gobernanza de datos y los componentes que se han asociado a su desarrollo, que se toman como marco analítico para realizar un análisis aplicado.

Para contrastar los aportes teóricos se plantea utilizar un estudio de caso, a partir de una investigación realizada con el Ayuntamiento de Barcelona, que permita identificar tanto aportes como retos planteados en las organizaciones públicas.

A través del estudio de caso como enfoque cualitativo se propone explorar un sistema acotado (la estrategia de gobernanza de datos impulsada por un gobierno local) mediante una detallada recopilación de datos con múltiples fuentes de información, y ofrecer una descripción analítica del objeto de estudio 
(los componentes de gobernanza de datos) atendiendo a los objetivos de la investigación (la relación entre gobernanza de datos e IA para asentar bases que propicien su desarrollo integral a nivel corporativo) (Creswell, 2018).

Con dicho propósito se plantea un estudio de caso orientado a la acción (action-oriented case study), un enfoque cualitativo con el que se pretende obtener información detallada sobre el contexto, los condicionantes, la estrategia y los procesos relacionados con los objetivos del estudio (Yin, 2018). La apuesta por el caso orientado a la acción se considera oportuna atendiendo al modelo de colaboración realizada entre un equipo investigador del que formó parte el autor y el Ayuntamiento de Barcelona, combinando reflexión conceptual con aportes relacionados con la práctica de la gestión pública local (Hult y Lennung, 1980; Huxham y Vangen, 2003).

A nivel más concreto, el proyecto se enmarcó en una colaboración entre el equipo investigador y el Ayuntamiento de Barcelona para realizar una reflexión estratégica y unas propuestas en materia de organización y modelos de gestión, que incluyó un apartado sobre inteligencia institucional que abordaba iniciativas vinculadas a la gobernanza de datos (Ramió y Salvador, 2019). El proyecto se desarrolló entre los meses de octubre de 2017 y noviembre de 2018 y combinó análisis documental (documentación interna), análisis de datos (sobre diferentes dimensiones de gestión) y la realización de 20 reuniones de seguimiento y contraste con un equipo multidisciplinar compuesto por directivos con amplio conocimiento de la institución. El formato de estas reuniones se basó en el contraste de reflexiones conceptuales y propuestas aplicadas a diferentes ámbitos de gestión, generando un diálogo abierto con el objetivo de construir conocimiento compartido. Adicionalmente se realizaron 15 entrevistas de apoyo tanto a actores internos como externos (12 con perfiles directivos y mandos de unidades internas vinculadas a la recopilación, análisis y difusión de datos; 3 con perfiles de consultoría especializada en tecnologías y diseño de cuadros de mando). Por el tipo de contenidos y modelo de colaboración no se grabaron las reuniones ni las entrevistas, pero se recopilaron las anotaciones realizadas (notas de campo e informes de resultado) por el equipo investigador que, con posterioridad a cada sesión, realizaba un diálogo reflexivo para discutir sus interpretaciones.

La aplicación del marco analítico definido a partir de los componentes de la gobernanza de datos y su vinculación con la IA al estudio de caso permite ofrecer resultados en clave de concreción de medidas impulsadas e identificar logros y retos pendientes en su desarrollo.

Para culminar el aporte se ofrecen unas conclusiones sobre los factores clave que inciden en la configuración de una estrategia de gobernanza de datos para el desarrollo de la IA en la administración pública.

\section{INTELIGENCIA ARTIFICIAL (IA) Y GOBERNANZA DE DATOS EN EL SECTOR PÚBLICO}

La literatura académica sobre la IA en el sector público constituye un campo en expansión, todavía de carácter incipiente, que suele centrar la atención en el surgimiento de soluciones para transformar servicios y políticas impulsadas por las organizaciones públicas, sus potenciales y sus riesgos.

En la relación entre IA y los datos cabe destacar los aportes tanto sobre sus potencialidades como sobre los requerimientos exigidos. Por un lado, la IA permite gestionar grandes bases de datos para mejorar el trabajo de los profesionales del sector público, en especial a partir de la integración de bases de datos internas y externas, incluso si incluyen información de diferente naturaleza (datos visuales, audios, espaciales, etc.), y tanto de carácter cuantitativo como cualitativo, para generar nuevos resultados (Mehr, 2017). Por otro lado, las necesidades de datos para la IA difieren de las requeridas por la analítica de datos tradicional. Para los procesos de auto-desarrollo, la IA requiere de ingentes cantidades de datos sobre los que basar sus dinámicas de interacción y aprendizaje. Por ello también resulta importante atender al tipo de datos que se proporcionan, puesto que el aprendizaje se basará en ellos (Susar y Aquaro, 2019; Gerbert et al., 2017). En la medida que se pretenda evitar sesgos o interpretaciones erróneas, es necesario que los científicos de datos tengan claramente definidos el tipo de datos a incorporar. Además, la IA conecta los datos a los procesos de acción y de aprendizaje en la medida que por un lado los utiliza para ofrecer resultados para decidir y para actuar ante una problemática considerada, pero al mismo tiempo utiliza dichos datos y resultados para su propio proceso de aprendizaje y mejora. Por ello resulta fundamental combinar datos, tecnología y procesos para generar sistemas integrados de IA en la organización.

Por ello, y como apuntan Wirtz et al. (2019), la IA será tan inteligentes como los datos de los que aprende. En este sentido resulta fundamental que los datos sean de calidad, íntegros y, especialmente, confia- 
bles. Para conseguir alinear IA y gestión de datos es necesario clarificar qué tipo de datos y evidencias son necesarias, su temporalidad y vigencia, dónde obtenerlos y como agregarlos para su adecuada utilización para generar procesos de IA (Mehr, 2017). La recopilación y agregación del tipo adecuado de datos resulta crítico para el éxito en este ámbito.

Otro reflejo de la creciente importancia otorgada a los datos es el surgimiento de una literatura académica vinculada a conceptos como el de «datificación», asociado a la digitalización y a la analítica del «big data», y que incluye las tecnologías y los procesos de trabajo a través de los cuales pueden gestionarse grandes cantidades de datos para monitorizar el comportamiento de determinadas variables (Loebbecke y Picot, 2015; Newell y Marabelli, 2015). Una línea de debate que se combina con aportes asociados tanto a la infraestructura que ha de facilitar sus procesos de obtención, almacenaje y tratamiento, como a las aplicaciones que han de permitir su gestión y su transformación, y a su utilización en los procesos de decisión y evaluación y sus consecuencias (Markus, 2017).

Aunque el concepto más extendido es el de «big data», que inicialmente se refería a aquellos datos digitales que son demasiado grandes, poco elaborados o desestructurados para ser analizados mediante técnicas convencionales de bases de datos relacionales, y que posteriormente evolucionó para incluir los procesos a través de los cuales las organizaciones obtienen valor de ellos (Guirguis, 2020; Kim et al., 2014). Una definición que, en el contexto del presente artículo, se asocia también a la acumulación de datos en repositorios, cuyo análisis mediante algoritmos propicia la toma de decisiones -sin que sea imprescindible la intervención humana-, generando procesos de transformación del trabajo, las organizaciones y la sociedad (Jones, 2019; Galliers et al., 2017).

En este contexto surge el concepto de gobernanza de los datos (data governance). Entre la multiplicidad de acepciones que aporta la literatura, Weber et al. (2009) y Khatri y Brown (2010) definen la gobernanza de datos en términos de marco que establece derechos y responsabilidades en la toma de decisiones sobre la gestión y el uso de datos. Otros aportes plantean entender el concepto como la confluencia de varias áreas relacionadas con los datos como los procesos para su administración, la gestión de su calidad o de su seguridad, entre otros (Begg y Caira, 2012). Más allá de la multiplicidad de aportes, existe cierto consenso en asociar la gobernanza de datos a las ideas de: (1) poner en valor los datos como un activo de la organización que debe gestionarse, (2) establecer responsabilidades en la toma de decisiones (derechos) y las tareas asociadas (deberes) y (3) establecer pautas y normas para velar por la calidad de los datos y su uso adecuado (Otto, 2011b). El concepto plantea el establecimiento de reglas del juego para cubrir el ciclo que incluye: recopilar, depurar y ordenar los datos, analizarlos e interpretarlos, elaborar informes y su visualización, ajustarlos y mejorarlos, y operacionalirzarlos e implementarlos. Siguiendo este argumento, la gobernanza de datos se asocia a los procesos organizativos que permiten especificar la asignación de responsabilidades y decisiones, alineadas con los objetivos de la organización, para impulsar un comportamiento deseable en el tratamiento de los datos como un activo de la organización (Otto, 2011a).

Planteada en estos términos, la gobernanza de datos se asocia al ámbito organizativo, focalizándose en cuestiones como el papel asignado al área de tecnologías, la centralización o descentralización de procesos y toma de decisiones o la definición de fórmulas de trabajo que convivan y atenúen tensiones organizativas. Una acepción organizativa que se enriquece al incorporar cómo la gobernanza de datos en el sector público plantea una serie de nuevos retos. Entre éstos cabe destacar el propio reconocimiento del valor de los datos (Nielsen et al., 2019; Begg y Caira, 2012), la vinculación de los niveles político y directivo con la gobernanza de los datos y el uso de sus resultados (Gonzalez-Zapata y Heeks, 2015), los condicionantes derivados de las cuestiones de seguridad y privacidad (Christodoulou et al. 2018), y los valores y la ética en el tratamiento y el desarrollo de soluciones basadas en los mismos (Pencheva et al., 2020).

En esta contextualización de los retos en el marco de las organizaciones del sector público deben tenerse en cuenta también como éstas suelen organizar sus datos (Jones, 2019; Kim et al., 2014). En parte como resultado del modelo burocrático que caracteriza a muchas entidades del sector, las administraciones públicas generan y recopilan grandes cantidades de datos, con diferentes finalidades y usos, con distintas tipologías y grados de estructuración, que suelen estar asociadas a diferentes departamentos que, a modo de silos, presentan importantes dificultades de integración. Por otro lado, y como se comentó anteriormente, los requerimientos de seguridad y salvaguarda de la privacidad cobran especial importancia atendiendo al contenido y naturaleza de los datos con los que operan las administraciones públicas.

Para afrontar esta multiplicidad de retos resulta fundamental atender a lo que la literatura vinculada a la IA refiere como condicionantes, requisitos o capacidades previas (o simultáneas, cuando menos). Con ello 
se hace referencia a la necesidad de contar con una infraestructura de recursos y competencias, y a utilizarla para desarrollar y optimizar la gestión y uso de datos para el desarrollo de soluciones de IA en el despliegue de su actividad.

A partir de las aportaciones de la literatura al respecto (Guirguis, 2020; Desouza et al., 2020; Keding, 2020; Susar y Aquaro, 2019; Ramió, 2019 y 2018; Desouza, 2018), en el presente artículo se propone interpretar estos condicionantes en términos de componentes de la gobernanza de datos que es necesario atender para su desarrollo y vinculación con la IA. Los componentes destacados son:

1. La estrategia, referida a la definición de una orientación sobre la gestión y uso de datos para el desarrollo de la IA. Una orientación que parte de la planificación y selección de los datos necesarios atendiendo a los retos que se plantean abordar. Ello implica tanto identificar qué datos son requeridos como qué fuentes de información deben conseguirse e integrarse. La disponibilidad de datos se convierte, para muchos ámbitos de política pública, en un reto a considerar en el desarrollo de soluciones de IA. Este direccionamiento estratégico debería incluir también las reglas del juego que fijan roles y relaciones tanto internas como externas, alrededor de la cadena de valor del dato, incluyendo cuestiones vinculadas a la actualización, acceso, disponibilidad, propiedad, seguridad, privacidad, y a los usos posteriores de los resultados obtenidos con los datos.

2. La arquitectura de datos (estándares, semántica, interoperabilidad) y la infraestructura de datos (almacenamiento y gestión de datos) para facilitar el desarrollo de los algoritmos y aplicaciones asociadas a la IA. Con ello se abordan cuestiones referidas a la generación de modelos que faciliten el intercambio de datos tanto interna como externamente, al mismo tiempo que se consideran aspectos como la escalabilidad o la infraestructura en red para para incrementar capacidades. En este ámbito también surgen debates como el de la internalización/externalización de determinadas infraestructuras. Aunque algunas organizaciones han apostado claramente por utilizar la nube (cloud) como recurso, en muchas administraciones públicas se sigue recurriendo a entornos locales, en aras de proteger una supuesta seguridad. Ello implica que deban planificarse los requerimientos de capacidad y de seguridad, tanto a corto como a medio y largo plazo, en especial si se incrementan exponencialmente los datos -con la incorporación de fuentes externas- y su analítica. Para dicho dimensionamiento también deben atenderse cuestiones como los datos a utilizar -como se generan y como se obtienen y procesan- o el tipo y cantidad de soluciones de IA que se plantean desarrollar.

3. La organización asociada a la gobernanza de datos, en referencia tanto a estructuras como procesos. Aquí se incluyen cuestiones como el equilibrio entre centralización y la descentralización de las diferentes actividades asociadas a la gestión y uso datos al servicio de la IA. Aunque las propuestas pueden variar atendiendo a la realidad de cada organización, en términos generales se apunta, por ejemplo, la conveniencia de centralizar repositorios de datos y procesos de diseño y aprendizaje de soluciones, lo que incluiría los procesos de preparación de los datos para su utilización, actividades de depuración, actualización o eliminación de errores, que resultan críticos para poder contar con base sólida sobre la que construir. De forma complementaria, se suele plantear la descentralización de las acciones tanto de identificación de preguntas y datos clave como de uso y aplicación de los resultados obtenidos. Un bloque en el que también se plantean cuestiones como la integración de lógicas transversales, superando los enfoques departamentales o sectoriales, y la incorporación de la flexibilidad en procesos y dinámicas de actuación para facilitar su mejora en el nuevo contexto.

4. La gestión del talento y las competencias de los profesionales de la organización. En este ámbito se destacan los perfiles profesionales que la organización debería potenciar para acompañar el diseño, la implantación y el desarrollo de la gobernanza de datos. La incorporación de analistas de datos, científicos de datos, desarrolladores, expertos en ciberseguridad, ingenieros de redes, profesionales de los sistemas de información, se suman a la renovación de capacidades técnicas y directivas para integrar los resultados en la estrategia de la organización. Para ello resulta clave invertir en la formación y en los procesos de cambio cultural asociados al desarrollo de la cultura de datos y su vinculación con la IA en la organización, incluyendo e implicando a los colectivos que actuarán como usuarios de la misma (en procesos de gestión y toma de decisiones).

5. El modelo relacional, referido al tipo de interacción tanto con el resto de organizaciones públicas como con las del sector privado y el sector social, así como con la ciudadanía. En el desarrollo de la gobernanza de datos vinculada a la IA se destaca la configuración de ecosistemas de entidades públicas y privadas que, a través del trabajo en red y la generación de sinergias, facilitan 
el avance y consolidación de transformaciones. La gestión de estas interacciones constituye otro de los componentes clave para el impulso de la gobernanza de datos en las organizaciones del sector público. En este componente destacan ideas como la posición de marca del ecosistema en su conjunto, el liderazgo en red, los procesos de intercambio (colaboración público-privada, externalización), y la generación de valor conjunto que va más allá de la mera suma de lo que aportan sus miembros individualmente.

La integración de los cinco componentes ofrece un marco analítico que permite revisar diferentes iniciativas aplicadas en el campo de la IA (actuales o planificadas), atendiendo especialmente a cómo se integra la gobernanza de datos como factor crítico para asentar las bases que faciliten su viabilidad.

\section{PRESENTACIÓN Y ANÁLISIS DE CASO: GOBERNANZA DE DATOS EN EL AYUNTAMIENTO DE BARCELONA}

A modo de contexto el Ayuntamiento de Barcelona constituye un referente reconocido internacionalmente en procesos de innovación y transformación digital (Ramió y Salvador, 2019), tanto en el ámbito social (Eizaguirre y Parés, 2019; Blanco et al., 2019) como en el de la promoción económica, la transformación urbana y la colaboración público-privada (Piqué et al., 2019; Acuto et al. 2018), también asociado al desarrollo de las smart cities (March y Ribera-Fumaz, 2018). Esta trayectoria y las interacciones entre las organizaciones públicas y privadas generaron un ecosistema urbano propicio a los procesos de innovación basados en la información y el conocimiento.

En este contexto el Ayuntamiento impulsó una serie de iniciativas y actuaciones vinculadas a la gobernanza de datos que se presentan y analizan en los siguientes sub-apartados, siguiendo el esquema analítico definido mediante los cinco componentes identificados en el apartado anterior.

\subsection{En relación a la estrategia}

Un reflejo de los aportes realizados en relación a este componente aparece en el propio plan de gobierno municipal donde se establecieron las «Medidas de gobierno para la digitalización abierta: software libre y desarrollo ágil de servicios a la Administración pública» que se plasmaron en el "Plan digital del Ayuntamiento de Barcelona 2017-2020" (http://ajuntament.barcelona.cat/imi/ca/memories/2016/el-pla-barcelonadigital-2017-2020). Un referente estratégico impulsado por la Comisionada de Tecnología e Innovación Digital (adscrita a la Primera Tenencia de Alcaldía) y la Comisión de Transformación Digital de Barcelona, que incluía el desarrollo de la estrategia de datos. La planificación vinculada a la estrategia Barcelona Ciudad Digital, se articulaba en tres ejes: Transformación Digital, Innovación Digital y Empoderamiento Digital (https://ajuntament.barcelona.cat/digital/es). El primero de ellos abordaba las cuestiones más vinculadas a los cambios internos y a la gestión de datos, incluyendo iniciativas sobre administración digital, infraestructura tecnológica urbana y el denominado «city data commons» (que incluía el Big Data para políticas públicas, el cuadro de mando municipal, o el portal de datos abiertos, entre otros).

Estos referentes explicitan una estrategia corporativa que incluye la gobernanza de datos, con un enfoque que pone el énfasis en la dimensión externa (empoderar a la ciudadanía), pero también el reconocimiento de la importancia de promover cambios internos, a nivel tecnológico, a nivel de instrumentos de apoyo al análisis (con el impulso de los cuadros de mando) y a nivel de cambio organizativo (con la creación de la Oficina Municipal de Datos, por ejemplo). Aunque estos referentes no llegaron a concretar la aproximación al gobierno del dato ni a clarificar cuestiones clave como las reglas del juego en su manejo, sí establecieron un marco que reflejaba un primer reconocimiento y fijaba las bases para el desarrollo de otros componentes clave.

\subsection{En relación a la arquitectura de datos y a la infraestructura}

La diversidad interna del Ayuntamiento se reflejaba también en sus dinámicas de denominación y generación de estándares para construir las bases de datos y alimentarlas. A través de la denominada Mesa de Coordinación Transversal de Datos (que se comenta más adelante) se abordaron estas cuestiones para generar normas compartidas que facilitasen el intercambio (interoperabilidad interna). 
Otro rasgo de la realidad tecnológica municipal era la multiplicidad de soluciones y aplicativos asociados a la producción, gestión, análisis y difusión de datos, reflejo de las lógicas organizativas diversas. A las mismas cabe añadir el solapamiento de soluciones tecnológicas adquiridas y desarrolladas en diferentes momentos y todavía en funcionamiento. A modo de ejemplos vinculados a la gestión y análisis de datos, destacaba el uso en diferentes departamentos de la aplicación Cognos, a modo de soporte para el desarrollo de aplicaciones de Business Intelligence (en especial cuadros de mando de apoyo a la toma de decisiones).

En paralelo al mantenimiento de ciertas aplicaciones propietarias, también se realizó una importante apuesta por herramientas de código abierto. Un ejemplo destacado es la plataforma Sentilo (https://connecta. bcn.cat/) vinculada a la recopilación, consulta y gestión de datos procedente de sensores distribuidos por la ciudad. Otro ejemplo es la plataforma digital Decidim Barcelona (https://www.decidim.barcelona/?locale=es), definida como portal e infraestructura tanto para los procesos de participación promovidos por el Ayuntamiento (Plan de Actuación Municipal, desarrollo de normas, planes de urbanismo, etc.) como para aquellos puestos en marcha por la ciudadanía y las asociaciones (iniciativas populares, desarrollo comunitario, etc.).

En lo referente a las transformaciones orientadas a generar soluciones compartidas a nivel corporativo, ya en clave de gobernanza de datos, cabe destacar la iniciativa de CityOs, una plataforma tecnológica de soluciones y servicios que permite la integración rápida, eficiente y fiable de los diferentes elementos de sensorización distribuidos por la ciudad y de las diferentes bases de datos y repositorios de información procedentes de múltiples fuentes y con formatos heterogéneos (http://ajuntament.barcelona.cat/imi/es/proyectos/cityos). Esta solución, promovida por la Oficina Municipal de Datos con el apoyo el Instituto Municipal de Informática, fue una respuesta orientada a facilitar la gestión del conocimiento de los diferentes servicios de la ciudad de una manera horizontal (entre servicios) y vertical (hasta un centro de supervisión global). Con ella se sentaban las bases de un enfoque más integrado para facilitar el desarrollo de soluciones de IA a nivel corporativo.

\subsection{En relación a la organización}

Un rasgo destacado de la realidad organizativa municipal referida a la gestión de datos y producción de información y conocimiento era la pluralidad de unidades con diferentes tipologías y roles. Entre éstas destacaban: (a) las unidades dedicadas a la gestión de datos y elaboración de informes para el conjunto del Ayuntamiento, incluyendo las vinculadas a ámbitos transversales o comunes como recursos humanos o gestión económica, entre otros, (b) las unidades dedicadas a la elaboración de informes y propuestas por ámbitos temáticos de carácter sectorial (por áreas o por territorios) y (c) la unidad central de apoyo tecnológico, el Instituto Municipal de Informática de Barcelona, que también actuaba como referente para la estrategia tecnológica y de transformación digital, incluyendo entre sus funciones la gobernanza del proceso de gestión de la información (https://ajuntament.barcelona.cat/imi/ca/memories/2017/linstitut-municipal-dinformatica).

Como iniciativa destacada para transformar esta realidad organizativa se creó, en febrero de 2018, la Oficina Municipal de Datos (OMD) (https://ajuntament.barcelona.cat/digital/es/transformacion-digital/city-data-commons/oficina-municipal-de-datos). Como Oficina del Dato (Magro, 2020; Wiseman, 2018), a la OMD se le asignó la responsabilidad de la gestión, la calidad, la gobernanza y la explotación de los datos en propiedad o custodiados por el Ayuntamiento de Barcelona y todos sus entes asociados (públicos o privados). Adscrita orgánicamente a la Gerencia Municipal, de la nueva oficina dependían las unidades vinculadas a la producción estadística propia -Departamento de Estadística-, de análisis -Gabinete Técnico de Programación-y la vinculada a la realización de encuestas y estudios de opinión. Desde esta unidad se impulsó la estrategia corporativa en materia de gestión de datos, estableciendo parámetros transversales para facilitar los procesos de diagnóstico y toma de decisiones. Esta labor de sistematización se realizó a través de la Mesa de Coordinación Transversal de Datos, con representación de perfiles directivos y técnicos de todas las áreas del Ayuntamiento, desde donde se resolvían cuestiones críticas como el modelo de meta-información o el despliegue de repositorios compartidos a nivel corporativo.

Además de la vertiente orgánica, al valorar el componente de la organización también deben considerarse los procesos y dinámicas desplegadas en relación a la gestión y utilización de datos. En este sentido cabe destacar experiencias previas como la impulsada en el Área de Ecología, Urbanismo y Movilidad (encargada de la prestación de los servicios municipales vinculados con el espacio público y los servicios urbanos como agua, espacios verdes, residuos o energía, y la vía pública), donde se desarrolló una Situation Room como plataforma que para visualizar y gestionar la información del funcionamiento de la ciudad en tiempo real a través de datos de los diferentes operadores que intervenían (https://ajuntament.barcelona.cat/ecologiaur- 
bana/ca/serveis/la-ciutat-es-transforma/resiliencia-urbana/analisi-informacio). La plataforma se basaba en datos cruzados entre todos los servicios municipales y del resto de los sistemas urbanos tanto públicos como privados, ofreciendo una cartografía y aportando conocimiento de apoyo para la toma de decisiones, tanto en el ámbito estratégico como operativo. Este ejemplo ilustra cómo los ámbitos más avanzados en la gestión de datos son aquellos que mediante sensores y otros mecanismos asociados de integración permiten una recopilación automatizada y el análisis de los mismos (movilidad, urbanismo, energía, residuos, etc.).

Pero también otros servicios que cuentan con otro tipo de recursos han impulsado sofisticados sistemas de información y de inteligencia institucional, como sería el caso de los servicios sociales (Informe Intel/ligència artificial: decisions automatitzades a Catalunya, 2020). Mediante una unidad de innovación, desde este ámbito se procedió a renovar la gestión de sus propios datos y a combinarlos con el big data de otras instituciones públicas (como la información tributaria en manos de la Administración General del Estado o los datos de las subvenciones sociales de la administración autonómica). Con esta información fueron capaces de identificar bolsas de pobreza, antes ocultas, en el seno de barrios acomodados que no eran atendidos por los servicios sociales municipales. Su utilización se extendió a cartografiar la ciudad de Barcelona, ubicando físicamente a las familias con problemáticas sociales y, de este modo, facilitar la reorganización de las redes estables y móviles de asistencia social. También desarrollaron actuaciones, a partir de la combinación de datos, para detectar fraudes en las ayudas sociales que prestaban las diferentes administraciones públicas.

A través de la Mesa de Coordinación Transversal de Datos antes citada se promovió la puesta en común de experiencias impulsadas por unidades tan diversas para facilitar tanto la puesta en valor de los datos como el desarrollo de iniciativas para impulsar su gestión y utilización en la toma de decisiones a nivel corporativo.

\subsection{En relación a la gestión del talento y las competencias}

A partir de un importante proceso de revisión del modelo de estructuración de su empleo público en 2018, el Ayuntamiento de Barcelona desplegó una configuración con ámbitos funcionales (como agrupación de perfiles profesionales) que incluían diversos vinculados al desarrollo de la gobernanza de datos como el de "servicios TIC», el de "archivo y documentación» y, especialmente, el de "servicios de análisis y estrategia» (https://bcnroc.ajuntament.barcelona.cat/jspui/bitstream/11703/112075/20/relacio_llocs_de_treball_bopb. pdf). A nivel más transversal, el modelo de empleo público del Ayuntamiento incorporaba un sistema de competencias profesionales con referencias también asimilables al ámbito de los datos como las de «búsqueda de información y actualización de conocimientos», "pensamiento analítico» o «flexibilidad y apertura al cambio», entre otras (https://bcnroc.ajuntament.barcelona.cat/jspui/bitstream/11703/105885/1/DicCom_catallotra_2012.pdf). Estas competencias se vinculaban al "Plan de formación y desarrollo 2016-2019" (https:// bcnroc.ajuntament.barcelona.cat/jspui/bitstream/11703/98671/1/Pla\%20de\%20formaci\%C3\%B3\%2020162019.pdf) que incluía acciones para promover la capacitación en ellas, además de ámbitos de actuación de carácter transversal como la formación en «gestión colaborativa, transversal y descentralizada» o en «administración electrónica y tecnologías de la información», entre otras. Pero en todo caso no se definieron explícitamente perfiles profesionales vinculados a la gobernanza datos ni se desarrolló una estrategia específica de desarrollo interno orientado a potenciar competencias vinculadas, y ni en las actuaciones de captación de nuevo talento ni en los procesos selectivos se priorizó particularmente esté ámbito de actuación.

\subsection{En relación al modelo relacional}

Un rasgo de la estrategia de transformación digital y de la Barcelona Ciudad Digital era su orientación externa. También en las funciones asignadas a la Oficina Municipal de Datos (OMD) se apuntaba la integración de diversidad de fuentes de datos (y no tan solo internas). Todo ello apunta a que desde el Ayuntamiento se promovió el desarrollo de enfoques colaborativos para propiciar redes de actores tanto públicos como privados, incluyendo un rol activo de la ciudadanía. La configuración de este ecosistema puso en valor el papel de la gobernanza de datos para optimizar las sinergias en clave de valor público.

En clave de colaboración con el entorno público cabe destacar, además lo comentado de intercambio de datos para determinadas iniciativas sectoriales, la participación conjuntamente con la Generalitat de Catalunya en organismos como el Centre of Innovation for Data tech and Artificial Intelligence (CIDAI, https:// cidai.eu/) o el BigDataCoE (Centro de Excelencia en Big Data de Barcelona (BigDATACoE, https://www. bigdatabcn.com/). 
En este entramado también surgieron fórmulas organizativas esencialmente privadas, pero en las que participaba indirectamente el Ayuntamiento, para promover actividades de producción y análisis de datos y conocimiento. Interpretadas como boundary-spanning organisations o BSO (Acuto et al., 2018), en el caso de Barcelona cabe destacar organismos impulsados desde la corporación municipal como el Urban i-Lab (https://ajuntament.barcelona.cat/digital/es/innovacion-digital/i-lab, para políticas de desarrollo local e innovación social) o Escolab (http://escolab.bcn.cat/, para el ámbito de la innovación, tecnología e innovación), u organismos impulsados desde la iniciativa privada -pero con presencia de la administración local- como la Fundación i2Cat (https://www.i2cat.net/\#home) para la promoción de la innovación con un componente tecnológico o el 22@Barcelona y la asociación 22@NetworkBcn (https://www.22network.net/associacio/) que agrupa organizaciones del distrito tecnológico del mismo nombre.

Finalmente cabe destacar la existencia de dinámicas de intercambio de información con empresas privadas, como las referenciadas para la iniciativa de la Situation Room del Área de Ecología, Urbanismo y Movilidad o del ámbito de servicios sociales.

Pero la diversidad de realidades apuntada inicialmente también se daba en los procesos de relación de colaboración en materia de datos con determinados sectores y empresas, suponiendo un reto especialmente importante cuando había servicios públicos implicados en ello. En muchos casos ello implicaba procesos de intercambio de datos poco eficaces y eficientes tanto con otras administraciones públicas como con determinados proveedores del propio Ayuntamiento, planteando un límite importante para consolidar una base a partir la cual construir soluciones integradas.

\section{DISCUSIÓN Y CONCLUSIONES}

Tal como se apuntaba en la introducción y en el diseño de la investigación, el artículo se proponía aportar reflexión y evidencias, a nivel conceptual y aplicado, sobre la relación entre la gobernanza de datos y la IA. Mediante la revisión del debate académico sobre la relación entre ambos conceptos se ha propuesto un esquema analítico basado en cinco componentes de la gobernanza de datos que pueden contribuir al desarrollo de la IA en el sector público. La aplicación de este esquema al estudio de caso del Ayuntamiento de Barcelona ha permitido tanto destacar sus potenciales y retos pendientes como ofrecer evidencias que permiten sustentar los argumentos apuntados y las conclusiones que se exponen a continuación.

El surgimiento de iniciativas sectoriales que utilizan la IA para la mejora de los servicios y las políticas públicas ofrece una imagen del potencial de este tipo de soluciones basadas en la tecnología. Por su carácter estratégico resulta fundamental que el sector público no quede ajeno a la integración de la IA en sus dinámicas de actuación. Para ello se plantea trascender de respuestas acotadas y de carácter puntual e integrar la IA en la lógica de funcionamiento de las administraciones públicas. Pero, ¿están preparadas estas organizaciones para asumir e integrar, a nivel transversal, la IA? Para sentar las bases de dicha integración se plantean una serie de propuestas que parten de la importancia de los datos y su gestión para ello.

La revisión conceptual que se ha ofrecido permite ilustrar, a través de los diferentes tipos de aportes realizados sobre la IA en el sector público, la importancia de los datos para su desarrollo. Sin embargo, su encaje resulta diferente del habitual en la analítica tradicional. Entre las diferencias que caracterizan el nuevo contexto cabe destacar el creciente volumen y diversidad de datos, la velocidad con la que se producen y actualizan, las nuevas conexiones entre ellos, unido a la sofisticación de las opciones analíticas que ofrece nuevas potencialidades. Pero el nuevo contexto implica también nuevos riesgos como la necesidad de revisar la veracidad de los datos, su calidad, su integridad y su trazabilidad, cuestiones vinculadas especialmente a su confiabilidad, como punto de partida para la obtención de resultados y soluciones válidas para la mejora de la actuación pública.

Para la extensión y desarrollo de la IA a nivel corporativo resulta esencial contar con nuevas capacidades que aborden la gestión y uso de los datos. En este contexto se destaca el concepto de gobernanza de datos, referida al establecimiento de las reglas del juego que han de regir el ciclo vinculado a la recopilación, depuración, ordenación, análisis e interpretación de datos, para facilitar la elaboración de resultados y su posterior utilización, implementación y evaluación de cara a su mejora.

Para la configuración de una gobernanza de datos vinculada al desarrollo de la IA en el sector público se han destacado cinco componentes que cubren desde la estrategia, la arquitectura e infraestructura de datos, la organización, la gestión del talento y las competencias, y el modelo relacional. La revisión de los 
aportes realizados a cada uno de los cinco componentes en el estudio de caso analizado del Ayuntamiento de Barcelona ofrece una visión de la importancia otorgada al ámbito y su combinación con la aproximación conceptual permite destacar algunos aprendizajes.

1. La importancia de establecer una estrategia a nivel corporativo que permita poner en valor la importancia de los datos y su adecuada gestión, incorporando las diferentes dimensiones de seguridad, privacidad, ética y responsabilidad. Pero que también incorpore una clara referencia a los componentes de la gobernanza de datos y su reflejo en las capacidades institucionales que han de hacer viable su desarrollo.

2. La articulación de la arquitectura y la infraestructura de datos para traducir, a nivel operativo, el valor del dato y su utilización, facilitando la consolidación de procesos de intercambio interno y externo que permitan generar sinergias a nivel corporativo y con el ecosistema de actores vinculados.

3. La asignación de responsabilidades y su reflejo orgánico, pero también a nivel de mecanismos de coordinación interna, con su reflejo en los procesos de gestión, que faciliten la consolidación de la gobernanza de datos, así como la implicación de las diferentes unidades y la generación de aprendizajes compartidos. La adecuada combinación de centralización y descentralización permite compartir este tipo de recursos y propiciar innovaciones adecuadas a diferentes realidades.

4. La traducción de la importancia de la gobernanza de datos en el ámbito de la gestión de recursos humanos, potenciando su reconocimiento en los mecanismos de selección, evaluación y retribución, como un valor que la organización reconoce y estimula. Aquí los retos son especialmente importantes debido a la distancia entre las lógicas y los ritmos de transformación propios de la gestión de los profesionales en el sector público en relación con los que se atribuyen a la gobernanza de datos. El peso de inercias y una diversidad de actores internos y externos con cierta aversión al riesgo y poco proclives a alterar los equilibrios vigentes, dificultan especialmente el cambio.

5. El modelo de relaciones con los actores presentes en el ecosistema de la gobernanza de datos y el desarrollo de la IA constituye otro de los componentes a replantear. Por ejemplo, potenciando las capacidades del sector público para establecer nuevos mecanismos de colaboración públicoprivada en los que se potencie el intercambio de datos con las empresas privadas implicadas, de modo dichos datos reviertan en repositorios para su posterior integración y utilización. O potenciar el seguimiento y evaluación de las externalizaciones para que, por ejemplo, la información generada revierta en el sector público que está financiando los servicios. O reforzando las capacidades de las administraciones públicas para generar iniciativas en clave de transparencia, rendimiento de cuentas y participación con las entidades sociales, pero también con la ciudadanía, fomentando su empoderamiento y su implicación responsable en el desarrollo de políticas públicas.

Los aportes concretos realizados a cada uno de los cinco componentes de la gobernanza de datos resultan importantes para sentar las bases de una IA a nivel corporativo. Pero para que dichos avances sean coherentes y puedan consolidarse resulta imprescindible que estén alineados con una estrategia clara, integrada y compartida para el desarrollo de este ámbito. El tipo de retos que plantea el despliegue de una gobernanza de datos, en la gestión y en la utilización de los mismos en las dinámicas de actuación y en los procesos de decisión, implican un cambio cultural profundo. Un factor intangible pero que se refleja claramente en actitudes y posiciones de los diferentes actores en relación al desarrollo de innovaciones como las que supone el imprescindible desarrollo de la IA en las administraciones públicas.

\section{REFERENCIAS BIBLIOGRÁFICAS}

Acuto, M., Steenmans, K., Iwaszuk, E. y Ortega-Garza, L. (2018). Informing urban governance? Boundary-spanning organisations and the ecosystem of urban data. Area, 51, 94-103. https://doi.org/10.1111/area.12430

Allam, Z. y Dhunny, Z. A. (2019). On big data, artificial intelligence and smart cities. Cities, 89, 80-91. https://doi. org/10.1016/j.cities.2019.01.032

Begg, C. y Caira, T. (2012). Exploring the SME Quandary: Data Governance in Practise in the Small to Medium-Sized Enterprise Sector. Electronic Journal of Information Systems Evaluation, 15(1), 3-13.

Nielsen, O. B., Persson, J. S. y Madsen, S. (2019). Why Governing Data Is Difficult: Findings from Danish Local Government. En A. Elbanna, Y. Dwivedi, D. Bunker y D. Wastell (eds.). Smart Working, Living and Organising. TDIT 2018. IFIP Advances in Information and Communication Technology (vol. 533). Springer. https://doi. org/10.1007/978-3-030-04315-5_2 
Blanco, I., Salazar, Y. y Bianchi, I. (2019). Urban governance and political change under a radical left government: The case of Barcelona. Journal of Urban Affairs, 42(1), 18-38. https://doi.org/10.1080/07352166.2018.1559648

Castro, D. y New, J. (10 de octubre de 2016). The promise of artificial intelligence. Center for Data Innovation. Consultado el 4 de agosto de 2020. https://www.datainnovation.org/2016/10/the-promise-of-artificial-intelligence/

Christodoulou, P., Decker, S., Douka, A. V., Komopoulou, C., Peristeras, V., Sgagia, S., Tsarapatsanis, V. y Vardouniotis, D. (2018). Data Makes the Public Sector Go Round. En P. Parycek, O. Glassey, M. Janssen, H. J. Scholl, E. Tambouris, E. Kalampokis, S. Virkar (eds.). Electronic Government. EGOV 2018. Lecture Notes in Computer Science (vol. 11.020). Springer. https://doi.org/10.1007/978-3-319-98690-6_19

Creswell, J. W. (2018). Qualitative inquiry and research design: choosing amōng five approaches. SAGE. https://doi. org/10.1177/1524839915580941

Desouza, K. C. (2018). Delivering Artificial Intelligence in Government: Challenges and Opportunities. IBM Center for the Business of Government. Consultado el 4 de agosto de 2020. http://www.businessofgovernment.org/report/ delivering-artificial-intelligence-government-challenges-and-opportunities

Desouza, K. C., Dawsona, G. S. y Chenok, D. (2020). Designing, developing, and deploying artificial intelligence systems: Lessons from and for the public sector. Business Horizons, 63(2), 205-213. https://doi.org/10.1016/j. bushor.2019.11.004

Eizaguirre, S. y Pares, M. (2019). Communities making social change from below. Social innovation and democratic leadership in two disenfranchised neighbourhoods in Barcelona. Urban Research \& Practice, 12(2), $173-191$. https://doi.org/10.1080/17535069.2018.1426782

Galliers, R. D., Newell, S., Shanks, G. y Topi, H. (2017). Datification and its human, organizational and societal effects: the strategic opportunities and challenges of algorithmic decision-making. Journal of Information Systems, 26(3), 185-190. https://doi.org/10.1016/j.jsis.2017.08.002

Gerbert, P., Hecker, M., Steinhäuser, S. y Ruwolt, P. (2017). Putting artificial intelligence to work. BCG Henderson Institute. Consultado el 4 de agosto de 2020. https://www.bcg.com/de-de/publications/2017/technologydigitalstrategy-putting-artificial-intelligence-work.aspx

Gonzalez-Zapata, F. y Heeks, R. (2015). The multiple meanings of open government data: Understanding different stakeholders and their perspectives. Government Information Quarterly, 32(4), 441-452. https://doi.org/10.1016/j. giq.2015.09.001

Grosz, B. J., Mackworth, A., Altman, R., Horvitz, E., Mitchell, T., Mulligan, D. y Shoham, Y. (2016). Artificial intelligence and life in 2030: One hundred years' study on artificial intelligence. Stanford University. Consultado del 4 de agosto de 2020. https://ai100.stanford.edu/sites/default/files/ai_100_report_0831fnl.pdf

Guirguis, K. (2020). From Big Data to Big Performance - Exploring the Potential of Big Data for Enhancing Public Organizations' Performance. A Systematic Literature Review. Swiss Yearbook of Administrative Sciences, 11(1), 55-65. https://doi.org/10.5334/ssas. 140

Jones, M. (2019). What we talk about when we talk about (big) data. The Journal of Strategic Information Systems, 28(1), 3-16. https://doi.org/10.1016/j.jsis.2018.10.005

Intel/ligència artificial: decisions automatitzades a Catalunya (2020). Barcelona: APDCAT - Generalitat de Catalunya. Consultado el 4 de agosto de 2020. https://apdcat.gencat.cat/web/.content/04-actualitat/noticies/documents/ INFORME-INTELLIGENCIA-ARTIFICIAL-FINAL-WEB-OK.pdf

Keding, C. (2020). Understanding the interplay of artificial intelligence and strategic management: four decades of research in review. Management Review Quarterly, 71, 91-134. https://doi.org/10.1007/s11301-020-00181-x.

Khatri, V. y Brown, C. V. (2010). Designing Data Governance. Communications of the ACM, 53(1), 148-152. https://doi. org/10.1145/1629175.1629210

Kim, G. H., Trimi, S. y Chung, J. H. (2014). Big Data Applications in the Government Sector: A Comparative Analysis among Leading Countries. Communications of the ACM, 57(3), 78-85. https://doi.org/10.1145/2500873

Loebbecke, C. y Picot, A. (2015). Reflections on Societal and Business Model Transformation Arising from Digitization and Big Data Analytics: A Research Agenda. The Journal of Strategic Information Systems, 24(3), 149-157. https:// doi.org/10.1016/j.jsis.2015.08.002

Luckin, R., Holmes, W., Griffiths, M. y Forcier, L. B. (2016). Intelligence Unleashed: An argument for Al in Education. Pearson Education. https://www.pearson.com/content/dam/corporate/global/pearson-dot-com/files/innovation/ Intelligence-Unleashed-Publication.pdf

Magro, R. (2020). La Oficina del Dato, El Consultor de los Ayuntamientos (Sección A Fondo), III, 99-110.

Markus, M. L. (2017). Datification, Organizational Strategy, and IS Research: What's the Score? The Journal of Strategic Information Systems, 26(3), 233-241. https://doi.org/10.1016/j.jsis.2017.08.003

Mehr, H. (agosto de 2017). Artificial intelligence for citizen services and government. Harvard Kennedy School, Ash Center for Democratic Governance and Innovation. Consultado el 4 de agosto de 2020. https://ash.harvard.edu/ files/ash/files/artificial_intelligence_for_citizen_services.pdf

Hult, M. y Lennung, S. $\bar{\AA}$. (1980). Towards a definition of action research: a note and bibliography. Journal of Management Studies, 17(2), 241-250. https://doi.org/10.1111/j.1467-6486.1980.tb00087.x

Huxham, C. y Vangen, S. (2003). Researching organizational practice through action research: case studies and design choices. Organizational Research Methods, 6(3), 383-403. https://doi.org/10.1177/1094428103254454 
March, H. y Ribera-Fumaz, R. (2018). Barcelona: from corporate smart city to technological sovereignty. En A. Karvonen, F. Cugurullo y F. Caprotti (eds). Inside Smart Cities: Place, Politics and Urban Innovation (págs. 227242). Routledge. https://doi.org/10.4324/9781351166201-15

Newell, S. y Marabelli, M. (2015). Strategic opportunities (and challenges) of algorithmic decision-making: a call for action on the long-term societal effects of «Datification». Journal of Strategic Information Systems, 24(1), 3-14. https://doi.org/10.1016/j.jsis.2015.02.001

Nikitas, A., Michalakopoulou, K., Njoya, E. T. y Karampatzakis, D. (2020). Artificial Intelligence, Transport and the Smart City: Definitions and Dimensions of a New Mobility Era. Sustainability, 12, 1-19. https://doi.org/10.3390/ su12072789

Otto, B. (2011a). A morphology of the organization of data governance. European Conference on Information Systems (ECIS 2011 Proceedings, 272). Association for Information Systems (AIS). https://aisel.aisnet.org/ecis2011/272

Otto, B. (2011b). Organizing Data Governance: Findings from the Telecommunications Industry and Consequences for Large Service Providers. Communications of the Association for Information Systems, 29, 45-66 (artículo 3). https://doi.org/10.17705/1cais.02903

Pencheva, I., Esteve, M. y Jankin Mikhaylov, S. (2020). Big Data and Al - A transformational shift for government: So, what next for research? Public Policy and Administration, 35(1), 24-44. https://doi.org/10.1177/0952076718780537

Pique, J. M., Miralles, F. y Berbegal-Mirabent, J. (2019). Areas of innovation in cities: the evolution of 22@Barcelona. International Journal of Knowledge-Based Development, 10(1), 3-25. https://doi.org/10.1504/ijkbd.2019.10019576

Qian Sun, T. y Medaglia, R. (2019). Mapping the challenges of Artificial Intelligence in the public sector: Evidence from public healthcare. Government Information Quarterly, 36(2), 368-383. https://doi.org/10.1016/j.giq.2018.09.008

Ramió, C. (2019). Inteligencia artificial y Administración pública. Robots y humanos compartiendo el servicio público. Catarata.

Ramió, C. (2018). El impacto de la inteligencia artificial y de la robótica en el empleo público. GIGAPP Estudios Working Papers, 5(98-110), 401-421. http://www.gigapp.org/ewp/index.php/GIGAPP-EWP/article/view/115

Ramió, C. y Salvador, M. (2019). Gobernanza social e inteligente. Una nueva organización para el Ayuntamiento de Barcelona. Ajuntament de Barcelona. https://ajuntament.barcelona.cat/barcelonallibres/sites/default/files/ publicacions_fitxers/pdf_gobernanza_indexat_cast.pdf

Weber, K., Otto, B. y Österle, H. (2009). One Size Does Not Fit All. A Contingency Approach to Data Governance. Journal of Data and Information Quality, 1(1), 1-26. https://doi.org/10.1145/1515693.1515696

Wiseman, J. M. (2018). Data-Driven Government: The Role of Chief Data Officers. IBM Center for the Business of Government. http://www.businessofgovernment.org/sites/default/files/Data-Driven\%20Government_0.pdf

Wirtz, B. W., Weyerer, J. C. y Geyer, C. (2019). Artificial Intelligence and the Public Sector - Applications and Challenges. International Journal of Public Administration, 42(7), 596-615. https://doi.org/10.1080/01900692.2018. 1498103

Yin, R. K. (2018). Case study research and applications: design and methods. SAGE. 